\title{
A multicenter study of a contrast-enhanced ultrasound diagnostic classification of breast lesions
}

This article was published in the following Dove Medical Press journal:

Cancer Management and Research

\section{Lina Tang, ',* Yijie Chen, ',* Zhongshi Du,' Zhaoming Zhong,' Qin Chen, ${ }^{2}$ Lichun Yang, ${ }^{3}$ Ruoxia Shen, ${ }^{3}$ Yan Cheng, ${ }^{4}$ Zizhen Zhang, ${ }^{4}$ Ehui Han, ${ }^{5}$ Zhihong Lv, ${ }^{5}$ Lijun Yuan, ${ }^{6}$ Yong Yang, ${ }^{6}$ Yinrong Cheng, ${ }^{7}$ Lei Yang, ${ }^{7}$ Shengli Wang, ${ }^{8}$ Baoyan Bai, ${ }^{8}$ Jun Luo ${ }^{2}$ \\ 'Department of Ultrasound, Fujian Cancer Hospital and Fujian Medical University Cancer Hospital, Fuzhou 3500 I4, Fujian Province, China; ${ }^{2}$ Department of Ultrasound, Sichuan Provincial People's Hospital, Chengdu 610072, Sichuan Province, China; ${ }^{3}$ Department of Ultrasound, The Third Affiliated Hospital of Kunming Medical University and Yunnan Cancer Hospital, Kunming 650II8, Yunnan Province, China; ${ }^{4}$ Department of Ultrasound, Qujing City First People's Hospital, Qujing 655000, Yunnan Province, China; ${ }^{5}$ Department of Ultrasound, Huangshi Central Hospital, Affiliated Hospital of Hubei Polytechnic University, Edong Healthcare Group, Huangshi 435000, Hubei Province, China; ${ }^{6}$ Department of Ultrasound, Tangdu Hospital, Fourth Military Medical University, Xi'an 710032, Shanxi Province, China; ${ }^{7}$ Department of Ultrasound, Chengdu First People's Hospital, Chengdu 610000, Sichuan Province, China; ${ }^{8}$ Department of Ultrasound Yanan University Affiliated Hospital, Yan'an \\ 7I6000, Shanxi Province, China \\ *These authors contributed equally to this work}

Correspondence: Jun Luo

Department of Ultrasound, Sichuan Provincia People's Hospital, 32 First Ring Road, Qingyang District, Chengdu 610072, China

Tel+86 I7740209986

Email milton-lj@hotmail.com

Lina Tang

Department of Ultrasound, Fujian Cance Hospital and Fujian Medical University Cancer Hospital, 420 Fuma Street, Fuzhou 350014,

China

Tel +86 I3625079386

Email tanglina@fjzlhospital.com
Purpose: To evaluate a classification model of contrast-enhanced ultrasound (CEUS) and examine the characteristics of patients with false-negative diagnosis.

Patients and methods: A retrospective secondary analysis of a multicenter trial of CEUS for breast cancer diagnosis (from August 2015 to April 2017) was undertaken. Patients $(n=1,023)$ with Breast Imaging Reporting and Data System 4-5 lesions on B-mode ultrasound underwent CEUS. Pathological diagnoses were available from surgical or biopsy specimens for correlation. Lesion maximum diameter (LMD), distance to the papilla (DtP), distance from the superficial edge of the lesion to the skin (DtS), distance from the deep edge of the lesion to the pectoralis muscle (DtPM), and body mass index (BMI) were evaluated.

Results: Median age and BMI were 48.0 and 41.2 years and 23.2 and $22.4 \mathrm{~kg} / \mathrm{m}^{2}$ for patients with malignant and benign lesions, respectively. Overall sensitivity, specificity, and accuracy of CEUS for malignancy were $89.4 \%, 65.3 \%$, and $75.8 \%$, respectively. The patients with truepositive and false-negative diagnosis (ie, with malignant lesion) were older than those with false-positive and true-negative diagnosis (ie, with benign lesion). Patients with true-positive and false-positive diagnoses had higher BMI than patients with true-negative and false-negative diagnoses $(P=0.004)$. Patients with true-positive and false-negative diagnoses had larger LMD and DtP, as well as smaller DtS and DtPM.

Conclusion: Older age, higher BMI, larger LMD and DtP, and smaller DtS and DtPM were associated with malignant lesions on CEUS. Patients with these characteristics should undergo further imaging.

Keywords: breast cancer, ultrasound, contrast-enhanced ultrasound

\section{Introduction}

Breast cancer is the most common cancer in women and the fifth cause of death from cancer. ${ }^{1}$ Breast cancer can be classified as invasive or in situ based on whether or not it invades the basement membrane of the duct of lobule. ${ }^{2}$ In the US, about $95 \%$ of the new cases of breast cancer occur in women $>40$ years of age. ${ }^{3}$ The worldwide incidence is about 43.3 new cases per 100,000 women, for a cumulative lifetime risk of $4.6 \% .^{3}$ In the US, mortality from breast cancer ranges from 11.3 to 30 cases per 100,000 , depending upon ethnic groups. ${ }^{4}$ Early diagnosis of breast cancer is primordial to enable appropriate treatments and improve prognosis. Many countries offer breast cancer screening programs, and these programs have been shown to decrease breast cancer mortality by $15 \%-30 \% .{ }^{5}$ On the other hand, screening is associated with risks of false-positive, false-negative, and over-diagnosis. ${ }^{6,7}$ Ultrasound is often used as an adjunct to mammography for screening to enable 
early diagnosis of breast cancer worldwide. An optimal screening tool should have both high sensitivity and specificity, but both mammography and B-mode ultrasound have sensitivity $<75 \% .{ }^{8}$ Newer and emerging technologies such as tomosynthesis, contrast-enhanced mammography, elastography, and contrast-enhanced ultrasound (CEUS) are promising. ${ }^{9-11}$

Ultrasound characterizes a breast lesion using morphological descriptors such as shape, margin, and orientation. In Western countries, ultrasound is used as an adjunct to mammography. In Asia, it is also used as adjunct to mammography, but the frequency of use is probably higher because Asian women have relatively small and dense breasts, complicating the interpretation of mammography. ${ }^{12,13}$ Therefore, this study aimed to determine whether CEUS could improve the diagnostic rate and hence reduce the numbers of unnecessary biopsies. Furthermore, compared with mammography, ultrasound requires a less complex setting, and is portable and inexpensive, making it a modality of choice for developing countries with hard-to-reach areas in Asia without a permanent setting for breast cancer screening. In many countries, breast lesions are categorized using the Breast Imaging Reporting and Data System (BI-RADS), in which the malignant risk of lesions with BI-RADS 4 ranges from $2 \%$ to $95 \%$, resulting in unnecessary invasive biopsy for many women. Raza et $\mathrm{al}^{14}$ showed that the positive predictive value of BI-RADS 4 lesions was only $15.5 \%-20.0 \%$.

Breast cancers typically have rich blood supply, ${ }^{15}$ making them candidates for the use of contrast-based imaging modalities. CEUS involves the use of a contrast agent confined to the vascular lumen and visible by ultrasound. CEUS can significantly improve the diagnostic accuracy of breast lesions compared to conventional ultrasound, in particular for BI-RADS $4 \mathrm{a}$ and $4 \mathrm{~b}$ lesions. ${ }^{16,17}$ Unfortunately, there is no unified classification criteria for CEUS analysis. Our team has previously established a CEUS classification based on six enhancement patterns, and this system has been shown to yield a diagnostic accuracy of $83.4 \%-86.4 \%$ in differentiating benign and malignant breast lesions. ${ }^{17}$

The aim of the present multicenter study was to further evaluate the performance of this model using a large cohort, and to examine the characteristics of patients with different classes of diagnosis (true negative, true positive, false negative, and false positive). The results could help inform about applicability of the model for breast cancer diagnosis.

\section{Methods}

\section{Study design and subjects}

This was a retrospective secondary analysis of a prospective multicenter (Fujian Cancer Hospital, Sichuan Provincial People's Hospital, Yunnan Cancer Hospital, Qujing City First People's Hospital, Huangshi Central Hospital, Tangdu Hospital, Chengdu First People's Hospital, and Yanan University Affiliated Hospital) trial of CEUS for breast cancer diagnosis, originally carried out between August 2015 and April 2017. The study has been approved by the ethics committee of Sichuan Provincial People's Hospital (No 2016 14-1). The patients provided written informed consent for the original study and any eventual secondary data analysis. The original study and this substudy were carried out according to the principles of the Declaration of Helsinki.

All centers that participated in the original trial have screening programs for breast cancer. Before enrollment, all patients underwent B-mode ultrasound. Only those $\geq 14$ years of age and with BI-RADS 4 and 5 breast lesions on B-mode ultrasound were recruited for the original CEUS trial. The exclusion criteria were: 1) contraindication or allergy to the contrast agent; 2) pregnant or breast-feeding; 3) coronary heart disease; 4) pulmonary or respiratory diseases; 5) any previous treatment for breast lesions (malignant or benign); 6) BI-RADS six lesions; or 7) diffuse lesions that cannot be measured or evaluated properly.

\section{B-mode ultrasound and CEUS}

The participating centers had different ultrasound systems (Philips iU22, Philips iU-Elite, Mylab 90, and GE LOGIQ E9), and each patient underwent ultrasound using a single system. B-mode ultrasound was performed with 5-12 MHz linear array transducers. CEUS was performed with 3-9 $\mathrm{MHz}$ linear transducers. The contrast agent used was SonoVue (Bracco SpA, Milan, Italy) reconstituted with $5 \mathrm{~mL}$ of sterile saline. The ultrasound parameters were: mechanical index $\leq 0.13 \mathrm{MHz}$, single focus at the bottom of the image, and probe stabilized manually without pressure.

The ultrasound physicians from each center had a minimum of 15 and 2 years experience in breast ultrasound and CEUS, respectively. The following parameters were measured and recorded: lesion maximum diameter (LMD), distance to the papilla (DtP) (Figure 1), distance from the superficial edge of the lesion to the skin (DtS) (Figure 2), and distance from the deep edge of the lesion to the pectoralis muscle (DtPM) (Figure 2).

During examination, the maximum imaging plane with rich blood supply or irregular shape of the mass including 


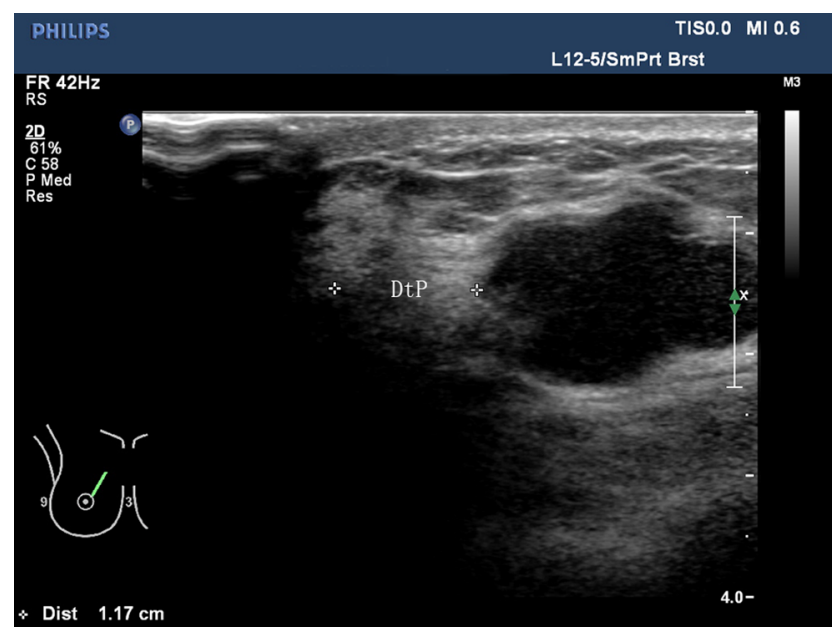

Figure I Measurement of the distance to the papilla (DtP). The distance shown in the figure is $1.17 \mathrm{~cm}$.

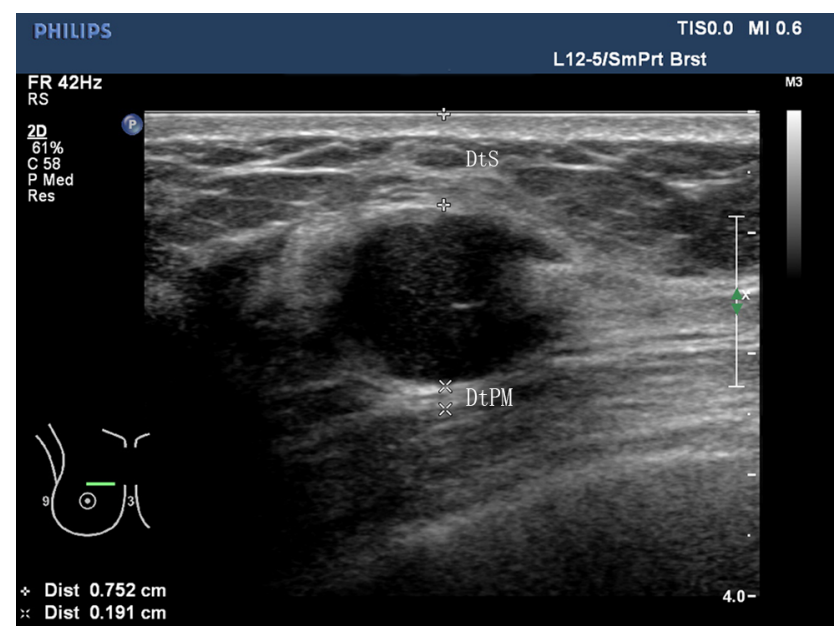

Figure 2 Measurement of the distance from the superficial edge of the lesion to the skin (DtS) and the distance from the deep edge of the lesion to the pectoralis muscle (DtPM).

Note: The distances shown in the figure are $D t S=0.752 \mathrm{~cm}$ and $D t P M=0.191 \mathrm{~cm}$.

its surrounding normal tissue was selected for CEUS. In the presence of multiple lesions in a single patient, only the largest one was evaluated by CEUS. After manual bolus injection of $4.8 \mathrm{~mL}$ of SonoVue via an antecubital vein, the selected plane was kept unchanged and real-time imaging was recorded for 2 minutes. All static and dynamic images were stored in the ultrasound systems and exported as JPEG and DICOM (digital imaging communications in medicine) files.

\section{Image analysis}

Image analysis was blinded and in accordance to the CEUS mode previously described. ${ }^{17}$ This classification consists of three benign (Figure 3) and three malignant (Figure 4)

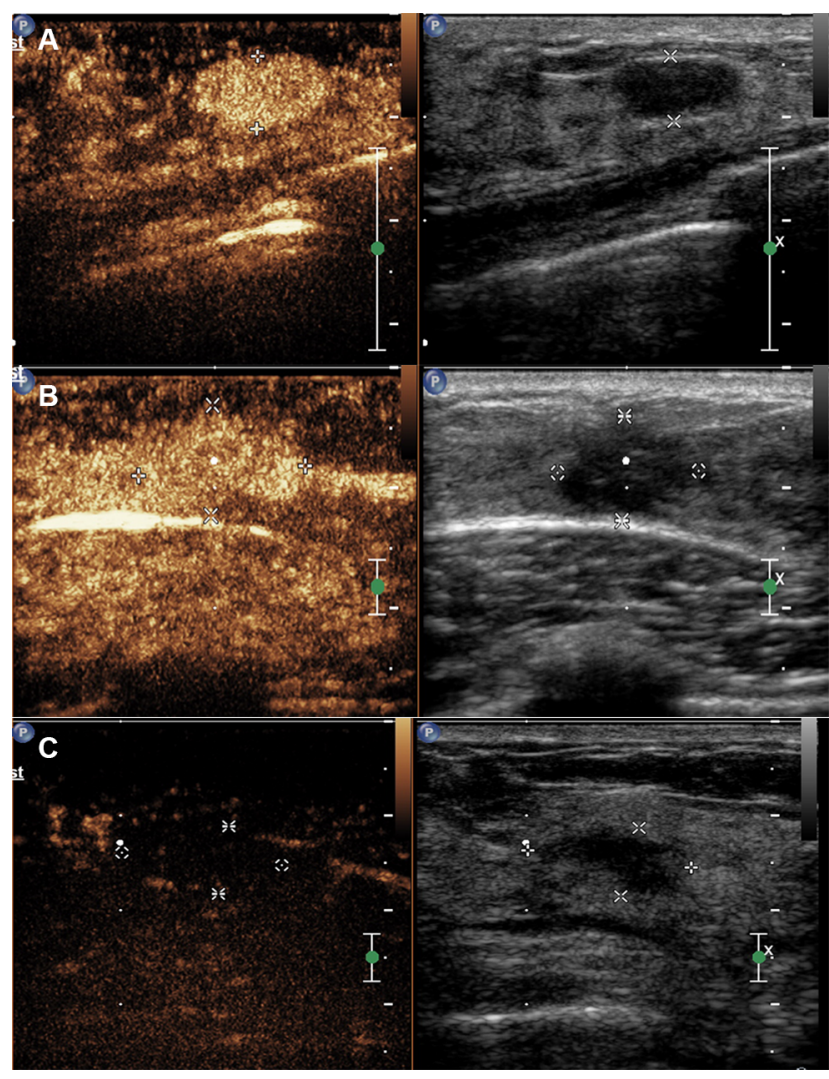

Figure 3 The benign sub-models of contrast-enhanced ultrasound for breast lesions. (A) Rapid wash-in with hyper-enhancement and clear margin after enhancement without enlarged size. (B) Synchronous or slow wash-in with iso-enhancement, and no difference in margin and shape after enhancement. (C) Synchronous or slow wash-in with hypo-enhancement.

classes: Figure 3 (A) hyper-enhancement with enlarged strength range, with or without irregular shape; B) hypercentripetal enhancement with perfusion defect, with or without enlarged range; C) rapid or synchronous wash-in with hyper- or iso-enhancement, presence of penetrating vessels or crab claw-like pattern, with or without perfusion defect; Figure 4A) rapid wash-in with hyper-enhancement, clear margin after enhancement without enlarged size; B) synchronous or slow wash-in with iso-enhancement, without distinction of margin and shape after enhancement; and C) synchronous or slow wash-in with hypo-enhancement.

The lesions that met any of the malignant or benign classes were categorized as malignant or benign. The lesions that met both of benign and malignant classes were categorized as malignant. Any disagreements were solved by the director of the ultrasound department of each center.

\section{Pathological data}

All patients underwent surgery or 16G core needle biopsy. The specimens were examined by two pathologists (at least 


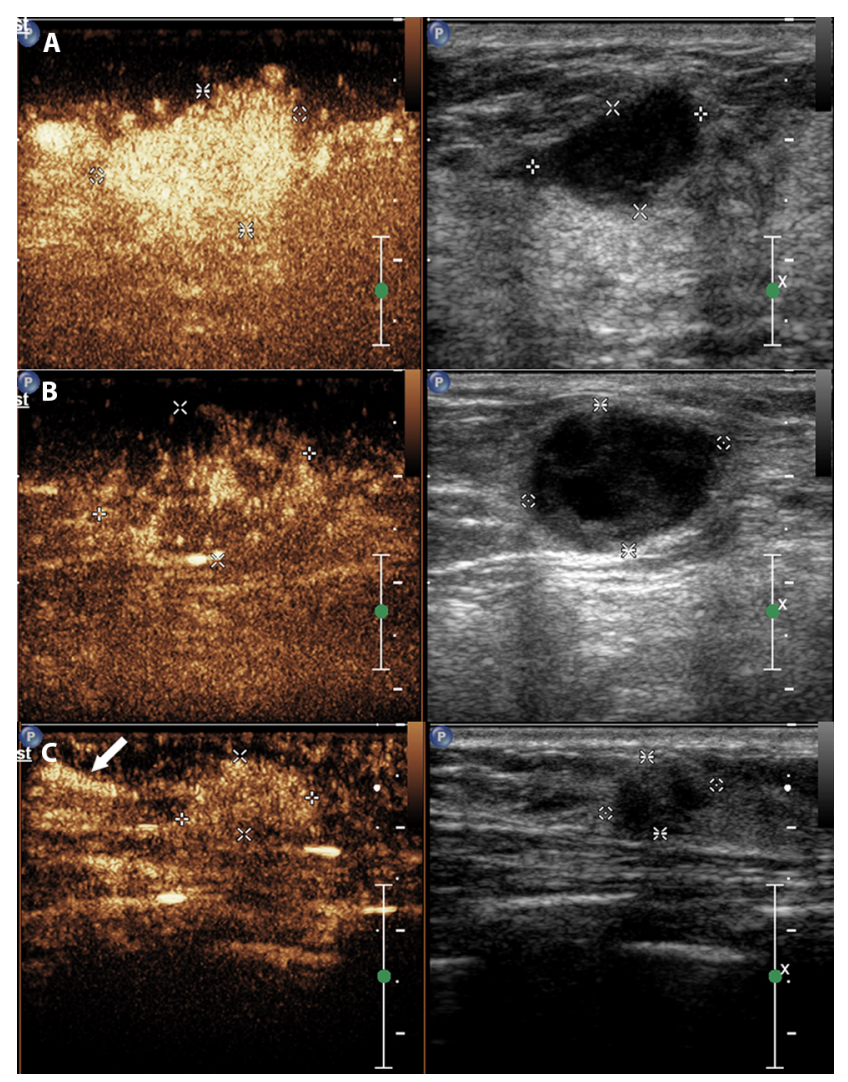

Figure 4 The malignant sub-models of contrast-enhanced ultrasound for breast lesions.

Notes: (A) Hyper-enhancement with enlarged range, with or without irregular shape. (B) Hyper-centripetal enhancement with perfusion defect, with or without enlarged range. (C) Rapid or synchronous wash-in with hyper- or iso-enhancement, presenting penetrating vessels (as indicated by the white arrows) or crab claw-like pattern, with or without perfusion defect.

3 years of experience in breast diagnosis) at each center. Diagnosis was based on the WHO criteria. ${ }^{2}$

\section{Diagnostic classification}

Based on the CEUS classification and the pathological diagnosis, each patient was classified as true positive, true negative, false positive, and false negative. Sensitivity, specificity, and accuracy were calculated.

\section{Data collection}

Age, body mass index (BMI; calculated as $\mathrm{kg} / \mathrm{m}^{2}$ ), number of lesions, lesion diagnosis, LMD, DtP, DtS, and DtPM were collected in the original trial.

\section{Statistical analysis}

Statistical analysis was conducted using SPSS 23.0 for Windows (IBM Corporation, Armonk, NY, USA). Continuous variables were analyzed using ANOVA (or the KruskalWallis test) and the Bonferroni's post hoc test. Categorical variables were analyzed with the chi-squared test. The null hypothesis was rejected at an $\alpha$ level of $5 \%(P<0.05)$.

\section{Results \\ Characteristics of the patients and lesions}

The study group included 1,023 female patients with 1,023 breast lesions evaluated by CEUS. Table 1 presents the characteristics of the patients and their lesions.

\section{Diagnostic performance of CEUS}

The overall sensitivity, specificity, and accuracy of CEUS for the diagnosis of breast lesions were $89.4 \%, 66.4 \%$, and $75.8 \%$, respectively.

\section{Characteristics of the lesions according to the diagnostic class}

Table 2 shows the characteristics of the lesions according to the diagnostic class. The patients with pathologically malignant lesions (true-positive and false-negative diagnoses) were older (true positive: 49.5 years; false negative: 47.2 years) than those with pathologically benign lesions (false positive: 41.2 years; true negative: 41.2 years) $(P<0.05)$. The patients with true-positive and false-positive diagnoses had a higher BMI (true positive: $23.1 \mathrm{~kg} / \mathrm{m}^{2}$; false positive: $\left.23.3 \mathrm{~kg} / \mathrm{m}^{2}\right)$ than patients with true-negative $\left(22.5 \mathrm{~kg} / \mathrm{m}^{2}\right)$ and false-negative $\left(22.4 \mathrm{~kg} / \mathrm{m}^{2}\right)$ diagnoses $(P=0.004)$. The patients with true-positive and false-negative diagnoses also had larger LMD and DtP, as well as smaller DtS and DtPM compared with patients with true-negative and false-positive diagnoses (LMD: 23.4 and 19.6 vs 14.8 and $17.1 \mathrm{~mm}$, respectively, $P<0.05$; DtP: 26.3 and 26.5 vs 20.4 and 23.1 mm, respectively, $P<0.05$; DtS: 5.9 and 4.8 vs 6.5 and 6.8 $\mathrm{mm}$, respectively, $P<0.05$; DtPM: 2.4 and 3.4 vs 3.8 and 4.4 $\mathrm{mm}$, respectively, $P<0.05$ ). These results suggest that lesions with false-negative diagnosis share the same characteristics than true-positive lesions, ie, high LMD and DtP, and small DtS and DtPM.

\section{Discussion}

CEUS has better accuracy than B-mode ultrasound for breast cancer diagnosis, but CEUS is limited by image analysis subjectivity. Therefore, this study aimed to evaluate the performance of a classification model of CEUS and examine the characteristics of patients with different classes of diagnosis (true positive, true negative, false negative, and false positive), in particular the characteristics of the patients with false-negative diagnosis. The results showed that the CEUS 
Table I Characteristics of the patients and lesions

\begin{tabular}{|c|c|c|c|c|}
\hline Variables & All, n (\%) & $\begin{array}{l}\text { Malignant } \\
\text { CEUS, n (\%) }\end{array}$ & $\begin{array}{l}\text { Benign } \\
\text { CEUS, n (\%) }\end{array}$ & $\begin{array}{l}\text { Malignant and } \\
\text { benign CEUS }{ }^{\mathrm{a}} \text {, n (\%) }\end{array}$ \\
\hline No (lesions) & 1,023 & 579 & 425 & 19 \\
\hline \multicolumn{5}{|l|}{ Age (years) } \\
\hline Median (range) & $44.7(|8-8|)$ & & & \\
\hline$\leq 35$ & $203(19.8)$ & $83(40.9)$ & $118(58.1)$ & $2(1.0)$ \\
\hline$>35$ & $820(80.2)$ & $496(60.5)$ & $307(37.4)$ & $17(2.1)$ \\
\hline \multicolumn{5}{|l|}{ Body mass index $\left(\mathrm{kg} / \mathrm{m}^{2}\right)$} \\
\hline Median (range) & $22.9(14.7-50.8)$ & & & \\
\hline$<25.0$ & $803(78.5)$ & $435(54.2)$ & $352(43.8)$ & $16(2.0)$ \\
\hline$\geq 25.0-29.9$ & $197(19.3)$ & $130(66.0)$ & $64(32.5)$ & $3(1.5)$ \\
\hline$\geq 30.0$ & $23(2.2)$ & $14(60.9)$ & $9(39.1)$ & 0 \\
\hline \multicolumn{5}{|l|}{ Histopathology } \\
\hline \multicolumn{5}{|l|}{ Benign lesions $(n=579)$} \\
\hline Fibroadenoma & $254(43.9)$ & $7 \mid(28.0)$ & $179(70.5)$ & $4(1.6)$ \\
\hline Fibrocystic mastopathy & $132(22.8)$ & $39(29.5)$ & $91(68.9)$ & $2(1.5)$ \\
\hline Complex sclerosing adenosis & $15(2.6)$ & $9(60.0)$ & $5(33.3)$ & $I(6.7)$ \\
\hline Hyperplasia & $72(12.4)$ & $16(22.2)$ & $56(77.8)$ & 0 \\
\hline Atypical hyperplasia & II (I.9) & $8(72.7)$ & $3(27.3)$ & 0 \\
\hline Granulomatous mastitis & $43(7.4)$ & $30(69.8)$ & $12(27.9)$ & $\mathrm{I}(2.3)$ \\
\hline Intraductal papilloma & $42(7.3)$ & $13(3 \mid .0)$ & $25(59.5)$ & $4(9.5)$ \\
\hline Benign phyllodes tumor & $9(1.6)$ & $3(33.3)$ & $6(66.7)$ & 0 \\
\hline Hamartoma & $\mathrm{I}(0.2)$ & 0 & $I(100)$ & 0 \\
\hline \multicolumn{5}{|l|}{ Malignant lesions $(n=444)$} \\
\hline IDC & $389(87.6)$ & $347(89.2)$ & $37(9.5)$ & $5(1.3)$ \\
\hline DCIS & $32(7.2)$ & $30(93.8)$ & $2(6.3)$ & 0 \\
\hline Mucinous carcinoma & II (2.5) & $7(63.6)$ & $2(18.2)$ & $2(1.8)$ \\
\hline Infiltrating lobular carcinoma & $4(0.9)$ & $3(75.0)$ & I (25.0) & 0 \\
\hline Diffused large B-cell lymphoma & $\mathrm{I}(0.2)$ & 0 & $I(100)$ & 0 \\
\hline Malignant phyllodes tumor & $3(0.7)$ & $2(66.7)$ & I (33.3) & 0 \\
\hline Solid neuroendocrine carcinoma & $\mathrm{I}(0.2)$ & $I(100)$ & 0 & 0 \\
\hline Sarcoma & $3(0.7)$ & 0 & $3(100)$ & 0 \\
\hline
\end{tabular}

Note: ${ }^{\text {TT }}$ The lesions that met both benign and malignant classes were categorized as malignant.

Abbreviations: DCIS, ductal carcinoma in situ; IDC, invasive ductal carcinoma.

Table 2 Characteristics of the patients according to the type of final diagnosis

\begin{tabular}{|c|c|c|c|c|c|}
\hline Factors & $\begin{array}{l}\text { True positive } \\
(n=397)\end{array}$ & $\begin{array}{l}\text { True negative } \\
(\mathrm{n}=378)\end{array}$ & $\begin{array}{l}\text { False positive } \\
(n=201)\end{array}$ & $\begin{array}{l}\text { False negative } \\
(\mathrm{n}=47)\end{array}$ & $P$-value \\
\hline Age (years) & $49.5(23-8 I)$ & $41.2(18-70)^{\mathrm{a}}$ & $41.2(20-72)^{b}$ & $47.2(18-79)^{e, f}$ & $<0.05$ \\
\hline Body mass index $\left(\mathrm{kg} / \mathrm{m}^{2}\right)$ & $23.11(14.7-50.8)$ & $22.47(16.0-43.9)^{\mathrm{a}}$ & $23.29(16.9-37.6)^{c}$ & $22.4(|6.5-3| .2)$ & 0.004 \\
\hline Lesion maximum diameter $(\mathrm{mm})$ & $23.35(5.0-75.5)$ & $14.78(4.0-55.8)^{\mathrm{a}}$ & $17.06(4-85)^{b, d}$ & $19.63(6.2-43.6)^{d}$ & $<0.05$ \\
\hline Distance to the papilla & $26.31(0-89)$ & $20.36(0-66)^{\mathrm{a}}$ & $23.13(0-76)$ & $26.51(0-80)$ & $<0.05$ \\
\hline Superficial edge of the lesion to the skin & $5.86(0-28)$ & $6.46(0-23)^{\mathrm{a}}$ & $6.80(0.30-23.0)^{\mathrm{b}}$ & $4.84(1-10)^{e, f}$ & $<0.05$ \\
\hline $\begin{array}{l}\text { Distance from the deep edge of the lesion to } \\
\text { the pectoralis muscle }\end{array}$ & $2.4 I(0-32)$ & $3.75(0-28)^{\mathrm{a}}$ & $4.35(0-31.8)^{\mathrm{b}}$ & $3.42(0-13)$ & $<0.05$ \\
\hline
\end{tabular}

Notes: All data are shown as median (range). ${ }^{\mathrm{a}}$ True positive vs true negative, $P<0.05$. ${ }^{\mathrm{b}}$ True positive vs false positive, $P<0.05$. ${ }^{\mathrm{c}}$ True negative vs false positive, $P<0.05$. ${ }^{\mathrm{d}}$ True negative vs false negative, $P<0.05$. ${ }^{\text {TT }}$ True positive vs false negative, $P<0.05$. ${ }^{\mathrm{f}} \mathrm{False}$ positive vs false negative, $P<0.05$.

classification for breast lesions is promising. Age, BMI, LMD, DtP, DtS, and DtPM were associated with diagnostic accuracy. Lesions with false-negative diagnosis share the same characteristics as true-positive lesions, ie, high LMD and DtP, and small DtS and DtPM. These results suggest that lesions with a negative diagnosis but with these specific characteristics should potentially be investigated more thoroughly since there is a risk of positive findings at pathologic examination. Nevertheless, there is also risk of false-positive diagnosis and this concern should be studied further.

Early diagnosis of breast diseases allows for earlier treatment and better prognosis. ${ }^{18}$ CEUS represents a method with 
good prospects for the early diagnosis of breast diseases and could be helpful to optimize the BI-RADS category. ${ }^{16,19}$ The enhancement patterns of lesions may be influenced by multiple factors such as microvessel density (MVD) and the intercellular substance structure. ${ }^{20}$ How to improve the diagnostic performance of CEUS is still an issue in the clinical application of CEUS. In the present study, the accuracy of the proposed CEUS classification for the diagnosis of malignant lesions was higher than that for benign lesions. It is noteworthy that the CEUS features of benign and malignant breast lesions can overlap. Model A is strongly predictive for malignancy, as confirmed in previous studies. ${ }^{19,21}$ Nevertheless, in the present study, $4.6 \%$ of the women had false-negative diagnosis, and many benign lesions also show enlarged size after enhancement. Our results showed that $72.7 \%$ of atypical hyperplasia lesions, $69.8 \%$ of granulomatous mastitis lesions, and $60.0 \%$ of complex sclerosing adenosis lesions were misdiagnosed. Further study on the enhancement features could help refine the models and improve accuracy.

In the present study, the overall sensitivity, specificity, and accuracy of CEUS for the diagnosis of breast lesions were $89.4 \%, 66.4 \%$, and $75.8 \%$, respectively. Sensitivity was similar to that reported by a meta-analysis of studies of CEUS, with $86 \%$ sensitivity, but the specificity was lower than in the meta-analysis (79\%). ${ }^{22}$ Similar observation can be made with B-mode ultrasound ( $87 \%$ sensitivity and $72 \%$ specificity) ${ }^{23}$ This could possibly be due to differences in image analysis and may suggest that our classification method could be improved. Nevertheless, the diagnostic performance of CEUS in the present study was similar to that of magnetic resonance imaging (92\% sensitivity and $70 \%$ specificity), but better than mammography in terms of sensitivity $(75 \%$ sensitivity and $71 \%$ specificity). ${ }^{24}$ Additional studies in various populations are necessary to determine the exact value of the classification system suggested in the present study.

Age is one of the most important risk factors since breast cancer in Western countries occurs around 55-70 years of age, ${ }^{4}$ but around $45-55$ years of age in Asian women. ${ }^{25}$ Thickness, duct width, and blood flow of the breast are different under the influence of hormones and according to the menopausal status. ${ }^{26}$ The results showed that patients with malignant lesions were older than those with benign lesions. Kim et a ${ }^{27}$ suggested that fibroadenoma in young women may enlarge rapidly and be accompanied by ductal epithelial hyperplasia, leading to increased number of peripheral microvessels, which may lead to false-positive CEUS results. Inflammatory responses could be accompanied by local vascular dilation and intraductal papilloma may display high enhancement, both potentially leading to false-positive CEUS diagnosis. ${ }^{28}$ Therefore, to reduce false-negative diagnosis in the clinical application of CEUS, the physicians should be aware that elderly patients are more likely to have malignant lesions, while young patients are more likely to have benign lesions, as in the present study. Nevertheless, the results of the present study should be interpreted in the context of our specific study population (Chinese women). The results should be confirmed in other populations around the globe.

Overweight or obese women have a higher risk of breast cancer compared with lean women. ${ }^{29}$ In the present study, patients with malignant lesions had a higher frequency of high BMI compared with women with benign lesions, and BMI was significantly different among the different diagnostic classes of CEUS. These results suggest that high BMI is associated with high risk of breast cancer as adipose tissue is considered as a major source of female hormones..$^{30}$ Indeed, after menopause, aromatase, the key enzyme in synthesis of estrogens, is mainly expressed in adipose tissue and aromatase inhibitors are part of the armament available against breast cancer. ${ }^{30}$

Lesion size is a crucial variable during ultrasound examination. It is highly associated with tumor angiogenesis, ${ }^{31}$ which is essential for tumor growth and infiltration. ${ }^{32}$ The strength of CEUS enhancement is closely related to MVD. ${ }^{33}$ In the present study, LMD was the largest in patients with true-positive CEUS diagnosis and the smallest in women with true-negative diagnosis, while those with false-positive and false-negative CEUS diagnosis had intermediate LMD values. This suggests that LMD is a major component of the qualitative analysis of CEUS images. In addition, some malignant lesions have no typical ultrasound features, neovascularization is not abundant, and heterogeneity is not obvious. ${ }^{28}$ It is difficult to determine the strength or type of enhancement of small lesions. As highlighted by Zhang et al, ${ }^{34}$ subjectivity plays a major role in determining whether there is heterogeneous enhancement of small lesions, and it may lead to high-false negative rates. For malignant lesions, with the enlargement of the lesion size, vessel heterogeneity is more prominent, spatial distribution is unbalanced, terminal fine vessels are increased, and tumor vessel branches are irregular. ${ }^{35}$ Nevertheless, taken together, these results suggest that large lesions should be at least considered suspicious, even in the presence of negative CEUS diagnosis.

DtP has been known for a long time to be associated with malignancy. DtP $>15 \mathrm{~mm}$ on mammography and ultrasound implies that the lesion is probably malignant. ${ }^{36}$ On the other hand, this feature has not been associated with malignancy 
for shear wave elastography, ${ }^{37}$ and there is no literature available regarding DtP in CEUS. In the present study, DtP of malignant lesions (true positive and false negative) was larger than for benign lesions. Additional studies are necessary to examine this feature in the context of CEUS.

Our results suggest that DtPM was smaller in malignant lesions (true positive and false negative) than in benign lesions (false positive and true negative). This could be explained by malignant nodules having larger diameter and longitudinal growth. In addition, DtS was smaller in malignant lesions than in benign lesions, but this parameter should be considered with caution because pressure from the transducer can greatly influence microcirculation perfusion, resulting in false-negative diagnosis. There can be important differences in DtS with ultrasound compared with mammography and MRI, possibly from the pressure exerted by the transducer on the breast. ${ }^{38,39}$ Although DtS is operator-dependent, it should be reproducible for different specific operators and could be used as a parameter for assessing malignancy.

There are some limitations to this study. Because this was a multicenter study, there was variability of equipment and physicians. The number of women $<35$ years of age was relatively small, and the value of CEUS in those patients still needs to be studied. No other imaging modality was used, and the value of the combination of multiple imaging techniques with CEUS needs to be examined. Finally, the use of different ultrasound systems is an important limitation of this study.

\section{Conclusion}

CEUS can synthesize multiple microvasculature and hemodynamic data of breast lesions. The rate of false-negative diagnosis could be influenced by age, BMI, LMD, DtP, DtS, and DtPM. The results support more features to improve the diagnostic performance of CEUS for breast lesions. Patients with false-negative diagnosis share the same characteristics as true-positive lesions, including high LMD and DtP, and small DtS and DtPM. These results suggest that patients with negative diagnosis but with these characteristics should possibly be investigated more thoroughly since there is a risk of positive findings. A larger international study including women of different nationalities is needed to validate this study.

\section{Acknowledgments}

The authors acknowledge the invaluable participation of the women as well as the help from the staff at all participating centers. The work was sponsored by Sichuan Provincial People's Hospital (\#2017HX001), Fujian Provincial Health
Technology Project (\#2015-1-11), and Science and Technology Program of Fujian Province, China (\# 2018Y2003).

\section{Disclosure}

The authors report no conflicts of interest in this work.

\section{References}

1. Bray F, Ferlay J, Soerjomataram I, Siegel RL, Torre LA, Jemal A. Global Cancer statistics 2018: GLOBOCAN estimates of incidence and mortality worldwide for 36 cancers in 185 countries. CA Cancer J Clin. 2018;68(6):394-424.

2. Lakhani SR, Ellis IO, Schnitt SJ, Tan PH, van de Vijver MJ. WHO Classification of Tumours of the Breast (IARC WHO Classification of Tumours). Lyon: IARC Press; 2012.

3. American Cancer Society. Breast Cancer - Facts \& Figures 2017-2018. Available from: https://www.cancer.org/content/dam/cancer-org/research/ cancer-facts-and-statistics/breast-cancer-facts-and-figures/breast-cancerfacts-and-figures-2017-2018.pdf. Accessed January 23, 2019.

4. Siegel RL, Miller KD, Jemal A. Cancer statistics, 2018. CA Cancer J Clin. 2018;68(1):7-30.

5. Njor S, Nyström L, Moss S, et al; Euroscreen Working Group. Breast cancer mortality in mammographic screening in Europe: a review of incidencebased mortality studies. J Med Screen. 2012;19(Suppl 1_):33-41.

6. Korhonen KE, Weinstein SP, McDonald ES, Conant EF. Strategies to increase cancer detection: review of true-positive and false-negative results at digital breast Tomosynthesis screening. Radiographics. 2016;36(7):1954-1965.

7. Shah TA, Guraya SS. Breast cancer screening programs: review of merits, demerits, and recent recommendations practiced across the world. J Microsc Ultrastruct. 2017;5(2):59-69.

8. Fiorica JV. Breast cancer screening, mammography, and other modalities. Clin Obstet Gynecol. 2016;59(4):688-709.

9. Covington MF, Pizzitola VJ, Lorans R, et al. The future of contrastenhanced mammography. AJR Am J Roentgenol. 2018;210(2):292-300.

10. Zhao YX, Liu S, Hu YB, Ge YY, Lv DM. Diagnostic and prognostic values of contrast-enhanced ultrasound in breast cancer: a retrospective study. Onco Targets Ther. 2017;10:1123-1129.

11. Botticelli A, Mazzotti E, Di Stefano D, et al. Positive impact of elastography in breast cancer diagnosis: an institutional experience. $J$ Ultrasound. 2015;18(4):321-327.

12. Rajaram N, Mariapun S, Eriksson M, et al. Differences in mammographic density between Asian and Caucasian populations: a comparative analysis. Breast Cancer Res Treat. 2017;161(2):353-362.

13. Ohuchi N, Suzuki A, Sobue T, et al; J-START investigator groups. Sensitivity and specificity of mammography and adjunctive ultrasonography to screen for breast cancer in the Japan strategic anti-cancer randomized trial (J-START): a randomised controlled trial. Lancet. 2016;387(10016):341-348.

14. Raza S, Goldkamp AL, Chikarmane SA, Birdwell RL. US of breast masses categorized as BI-RADS 3, 4, and 5: pictorial review of factors influencing clinical management. Radiographics. 2010;30(5):1199-1213.

15. LiYJ, Wen G, Yang L, Zhang X-Lin. [Heterogeneity of angioarchitecture and their hemodynamic changes in benign and malignant breast tumors]. Zhonghua Zhong Liu Za Zhi. 2009;31(1):24-27. Chinese.

16. Wan C, Du J, Fang H, Li F, Wang L. Evaluation of breast lesions by contrast enhanced ultrasound: qualitative and quantitative analysis. Eur J Radiol. 2012;81(4):e444-e450.

17. Luo J, Chen JD, Chen Q, et al. Predictive model for contrast-enhanced ultrasound of the breast: is it feasible in malignant risk assessment of breast imaging reporting and data system 4 lesions? World J Radiol. 2016;8(6):600-609.

18. Caplan L. Delay in breast cancer: implications for stage at diagnosis and survival. Front Public Health. 2014;2(2):87. 
19. Liu H, Jiang YX, Liu JB, Zhu QL, Sun Q. Evaluation of breast lesions with contrast-enhanced ultrasound using the microvascular imaging technique: initial observations. Breast. 2008;17(5):532-539.

20. Li X, Li Y, Zhu Y, Fu L, Liu P. Association between enhancement patterns and parameters of contrast-enhanced ultrasound and microvessel distribution in breast cancer. Oncol Lett. 2018;15(4):5643-5649.

21. Xiao X, Ou B, Yang H, Wu H, Luo B. Breast contrast-enhanced ultrasound: is a scoring system feasible? A preliminary study in China. PLoS One. 2014;9(8): 105517.

22. Hu Q, Wang XY, Zhu SY, Kang LK, Xiao YJ, Zheng HY. Meta-analysis of contrast-enhanced ultrasound for the differentiation of benign and malignant breast lesions. Acta Radiol. 2015;56(1):25-33.

23. Li Q, Hu M, Chen Z, et al. Meta-analysis: contrast-enhanced ultrasound versus conventional ultrasound for differentiation of benign and malignant breast lesions. Ultrasound Med Biol. 2018;44(5):919-929.

24. Zhang Y, Ren H. Meta-analysis of diagnostic accuracy of magnetic resonance imaging and mammography for breast cancer. $J$ Cancer Res Ther. 2017;13(5):862-868.

25. Chen W, Zheng R, Baade PD, et al. Cancer statistics in China, 2015. CA Cancer J Clin. 2016;66(2):115-132.

26. Denholm R, De Stavola B, Hipwell JH, et al. Pre-natal exposures and breast tissue composition: findings from a British pre-birth cohort of young women and a systematic review. Breast Cancer Res. 2016;18(1):102.

27. Kim SJ, Park YM, Jung SJ, et al. Sonographic appearances of juvenile fibroadenoma of the breast. J Ultrasound Med. 2014;33(11):1879-1884.

28. Zhang J. Breast Ultrasound Diagnosis. Beijing: People's Medical Publishing House; 2012.

29. Gathirua-Mwangi WG, ZollingerTW, Murage MJ, Pradhan KR, Champion VL. Adult BMI change and risk of breast cancer: National Health and Nutrition Examination Survey (NHANES) 2005-2010. Breast Cancer. 2015;22(6):648-656.

30. Ahn J, Schatzkin A, Lacey JV, et al. Adiposity, adult weight change, and postmenopausal breast cancer risk. Arch Intern Med. 2007;167(19): 2091-2102.
31. Lai HW, Chen DR, Wu YC, et al. Comparison of the diagnostic accuracy of magnetic resonance imaging with sonography in the prediction of breast cancer tumor size: a concordance analysis with histopathologically determined tumor size. Ann Surg Oncol. 2015;22(12): 3816-3823.

32. Goubran HA, Kotb RR, Stakiw J, Emara ME, Burnouf T. Regulation of tumor growth and metastasis: the role of tumor microenvironment. Cancer Growth Metastasis. 2014;7:9-18.

33. Wan CF, Du J, Fang H, Li FH, Zhu JS, Liu Q. Enhancement patterns and parameters of breast cancers at contrast-enhanced us: correlation with prognostic factors. Radiology. 2012;262(2):450-459.

34. Zhang JX, Cai LS, Chen L, Dai JL, Song GH. CEUS helps to rerate small breast tumors of BI-RADS category 3 and category 4. BioMed Res Int. 2014;2014(4):1-8.

35. Golbabapour S, Pang WW, George J, Pasupati T, Abdul-Rahman PS, Hashim $\mathrm{OH}$. Chemically induced breast tumors in rats are detectable in early stages by contrast enhanced magnetic resonance imaging but not by changes in the acute-phase reactants in serum. Int $J \mathrm{Mol} \mathrm{Sci}$. 2011;12(2):1030-1040.

36. El Sheikh H, Almetaher HA, Abdalla HS, Shareef MM. Intraductal breast masses: sonographic and mammographic predictors of malignancy. The Egyptian Journal of Radiology and Nuclear Medicine. 2015;46(4):1271-1278.

37. Chen YL, Gao Y, Chang C, Wang F, Zeng W, Chen JJ. Ultrasound shear wave elastography of breast lesions: correlation of anisotropy with clinical and histopathological findings. Cancer Imaging. 2018; 18(1):11.

38. Telegrafo M, Rella L, Stabile Ianora AA, Angelelli G, Moschetta M. Supine breast us: how to correlate breast lesions from prone MRI. $\mathrm{Br}$ J Radiol. 2016;89(1059):20150497.

39. Park JM, Yang L, Laroia A, Franken EA, Fajardo LL. Missed and/or misinterpreted lesions in breast ultrasound: reasons and solutions. Can Assoc Radiol J. 2011;62(1):41-49.
Cancer Management and Research

\section{Publish your work in this journal}

Cancer Management and Research is an international, peer-reviewed open access journal focusing on cancer research and the optimal use of preventative and integrated treatment interventions to achieve improved outcomes, enhanced survival and quality of life for the cancer patient. The manuscript management system is completely online and includes

\section{Dovepress}

a very quick and fair peer-review system, which is all easy to use. Visit http://www.dovepress.com/testimonials.php to read real quotes from published authors. 